\title{
Fuzzy Space-Time
}

\author{
J. Madore \\ Laboratoire de Physique Théorique et Hautes Energies* \\ Université de Paris-Sud, Bât. 211, F-91405 Orsay
}

\begin{abstract}
A review is made of recent efforts to define linear connections and their corresponding curvature within the context of noncommutative geometry. As an application it is suggested that it is possible to identify the gravitational field as a phenomenological manifestation of space-time commutation relations and to thereby clarify its role as an ultraviolet regularizer.
\end{abstract}

LPTHE Orsay 96/64

*Laboratoire associé au CNRS, URA D0063 


\section{Motivation}

Simply stated, noncommutative geometry is geometry in which the 'coordinates' do not commute. Since they cannot therefore be simultaneously diagonalized, points are ill-defined. This is a fact which is familiar from quantum mechanics; the canonical commutation relations lead to the Heisenberg uncertainty relations. The set of functions on an ordinary space form a commutative algebra. Under certain conditions it can be considered as an algebra generated by the coordinates. In noncommutative geometry this algebra is replaced by a noncommutative algebra which cannot be an algebra of functions; the algebra remains but the space disappears. The geometry of ordinary smooth spaces was written from the point of view of the algebra of smooth functions by Koszul (1960) in his lectures at the Tata Institute.

There are three more-or-less independent arguments in favour of the use of noncommutative geometry in high-energy physics and field theory. The first is of a very practical nature. When a physicist calculates a Feynman diagram he is forced to place a cut-off $\Lambda$ on the momentum variables in the integrands. This means that he renounces any interest in regions of space-time of dimension less than $\Lambda^{-1}$. As $\Lambda$ becomes larger and larger the forbidden region becomes smaller and smaller but it can never be made to vanish. There is a fundamental length scale, of the order of the Planck length, below which the notion of a point is of no practical importance. The cut-off must be bounded by the Planck mass. The simplest and most elegant, if certainly not the only, way of introducing such a scale in a Lorentz-invariant way is through the introduction of non-commuting coordinates.

A closely related way of stating the same thing is to say that the standard description of Minkowski space as a 4-dimensional continuum is redundant. There are too many points. It was proposed already by Heisenberg in the early days of quantum field theory to replace the continuum by a lattice structure. A lattice however breaks Poincaré invariance and can hardly be considered as fundamental. It was Snyder (1947) who first had the idea of using non-commuting coordinates to mimic a discrete structure in a covariant way. Since then several attempts have been made to continue this initial effort. One typically replaces the four Minkowski coordinates $x^{\mu}$ by four generators $q^{\mu}$ of a noncommutative algebra $\mathcal{A}_{\mu_{P}}$ which satisfy commutation relations of the form

$$
\left[q^{\mu}, q^{\nu}\right]=i \mu_{P}^{-2} q^{\mu \nu}
$$

The problem lies then with the interpretation of the right-hand side. The parameter $\mu_{P}$ is the Planck mass. One of our purposes is to discuss the physical significance of $q^{\mu \nu}$ and its relation to the gravitational field in the commutative limit.

As a simple example of a space one can consider the ordinary round 2-sphere which has acting on it the rotational group $\mathrm{SO}_{3}$. As a simple example of a lattice structure one can consider two points on the sphere. One immediately notices of course that by choosing the two points one has broken the rotational invariance and that however it can be restored by admitting noncommuting 'coordinates'. The set of functions on the two points can be identified with the algebra of diagonal $2 \times 2$ matrices, each of the two entries on the diagonal corresponding to a possible value of a function at one of the two points. Now an action of a group on a space is equivalent to an action of the group on the algebra of functions on the space. There can obviously be no (non-trivial) action of the group $\mathrm{SO}_{3}$ on the algebra of diagonal $2 \times 2$ matrices but if one extends the algebra to the noncommutative algebra of all $2 \times 2$ matrices one recovers the invariance. The two points, so to speak, have been smeared out over the surface of a sphere. They are replaced by two Bohr-like fuzzy regions. Although what we have just done has nothing 
to do with Planck's constant it is similar to the procedure of replacing a classical spin which can take two values by a quantum spin of total spin $1 / 2$. Only the latter is invariant under the rotation group. By replacing the spin $1 / 2$ by arbitrary spin $s$ one can (Madore 1992) describe a 'lattice structure' of $2 s+1$ points in an $\mathrm{SO}_{3}$-invariant manner. On this structure the notion of vectors and covectors can be introduced as well as the noncommutative generalization of a metric and linear connection. It can be readily shown that the unique torsion-free metric connection is expressible in terms of the structure constants of the Lie algebra of $\mathrm{SO}_{3}$. We would like to examine to what extent this result can be extended to noncommutative versions of Minkowski space, that is to what extent the commutation relations which restrict the algebra determine the possible gravitational fields which can be put on it. By 'gravitational field' we shall mean linearized 'gravity' in a noncommutative version of Minkowski space.

It is an old idea, due to Pauli and developed by Deser (1957) and others (Isham et al. (1971), that perturbative ultraviolet divergences will one day be regularized by the gravitational field. The possibility which we would like to explore here is that the mechanism by which this works is through the introduction of noncommuting "coordinates' such as the $q^{\lambda}$. A hand-waving argument can be given (Madore \& Mourad 1995) which allows one to think of the noncommutative structure of space-time as being due to quantum fluctuations of the light-cone in ordinary 4-dimensional space-time. This relies on the existence of quantum gravitational fluctuations. A purely classical argument based on the formation of black-holes has been given by Doplicher et al. (1994, 1995). In both cases the classical gravitational field is to be considered as regularizing the ultraviolet divergences through the introduction of the noncommutative structure of space-time. This can be strengthened as the conjecture that the classical gravitational field and the noncommutative nature of space-time are two aspects of the same thing; they are both measures of the spectral densities of the operators $q^{\lambda}$. The metric connection of the 'fuzzy-sphere' example mentioned above has a constant curvature and the spectral density of each coordinate is uniform. One could say, inexactly but suggestively, that the classical gravitational field arises from the 'first quantization' of the coordinates. We use here the word 'quantum' in the loose way which implies that something does not commute. It has nothing a priori to do with the 'quantum' of quantum mechanics.

It is to be stressed that we modify the structure of space-time but maintain covariance under the action of the Poincaré group. A fuzzy space-time looks then like a solid which has a homogeneous distribution of dislocations but no disclinations. We can pursue this solid-state analogy and think of the ordinary Minkowski coordinates as macroscopic order parameters obtained by coarse-graining over scales less than the fundamental scale. They break down and must be replaced by elements of the algebra when one considers phenomena on these scales. Another definition of 'quantum space-time' implies that it is covariant under the (co-)action of a 'quantum' Poincaré group.

A linguistic digression is in order. When referring to the version of space-time which we describe here we use the adjective 'fuzzy' to underline the fact that points are illdefined (Madore 1992, 1995). Since the algebraic structure is described by commutation relations the qualifier 'quantum' has also been used (Snyder 1947, Doplicher et al. 1995, Madore \& Mourad 1996b). This latter expression is unfortunate since the structure has no immediate relation to quantum mechanics and also it leads to confusion with 'spaces' on which 'quantum groups' act. To add to the confusion the adjective 'fuzzy' has been also used (Frittelli et al. 1996) to describe a rather different noncommutative structure and the word 'lattice' has been used ('t Hooft 1996) to designate what we 
here qualify as 'fuzzy'.

In Section 2 we describe in more detail the basic algebra which we shall suppose to replace the continuum description of Minkowski space. The use of noncommutative geometry leads of course to many problems. One must introduce the noncommutative generalization of a vector and a covector. This is done in Sections 3 and 4 . The gravitational field is described by a metric and a linear connection. In Section 5 the noncommutative generalizations of these objects are recalled. There is at the moment no completely satisfactory definition of the curvature of a noncommutative linear connection in all generality but in the particular cases which shall interest us here the usual definition in terms of the covariant derivative will suffice. We are unable at the moment to propose a satisfactory definition of an action and indeed we are not in a position to argue that there is even a valid action principle. A discussion of this point has been made by Connes and coworkers in a series of articles (Kalau \& Walze 1995, Ackermann \& Tolksdorf 1996, Connes 1996, Chamseddine \& Connes 1996) but the definition which these authors propose is valid only on the noncommutative generalizations of compact spaces with euclidean-signature metrics. More technical details of the present calculations are to be found in the article by Madore \& Mourad (1996b).

\section{Fuzzy space-time}

We start with an algebra $\mathcal{A}_{\mu_{P}}$ with 4 generators $q^{\lambda}$ which satisfy the commutation relations (1.1). Since we wish the limit space-time to be real we must suppose that the algebra has a $*$-operation which replaces the complex conjugate and that the $q^{\lambda}$ are hermitian. If we wished to do serious analysis we would have to add a topology to $\mathcal{A}_{\mu_{P}}$. Our first problem is the interpretation of the right-hand side of (1.1). It is the value of the commutator $\left[q^{\lambda}, q^{\mu \nu}\right]$ which restricts the structure of the algebra. One possibility, considered by Snyder (1947), is to choose it so that the algebra closes to form a representation of the Lie algebra of the de Sitter group. A second possibility, considered by Dubois-Violette \& Madore (Madore 1988, 1995) is to choose it so that the algebra closes to form a representation of the conformal algebra. We refer to Madore \& Mourad (1996a) for a review with historical perspective. Recently Doplicher, Fredenhagen \& Roberts $(1994,1995)$ have argued that $q^{\mu \nu}$ should in fact be considered as a new geometric quantity and that it can be chosen to lie in the center of $\mathcal{A}_{\mu_{P}}$. We shall follow this suggestion here and set therefore as a first approximation

$$
\left[q^{\lambda}, q^{\mu \nu}\right]=0
$$

We shall see however that in order to introduce non-trivial gravitational fields we shall be lead naturally to a non-vanishing term on the right-hand side. We shall suppose that the limit $\mathcal{A}_{0}$ of the algebra $\mathcal{A}_{\mu_{P}}$ when $\mu_{P} \rightarrow \infty$ exists and we set

$$
x^{\lambda}=\lim _{\mu_{P} \rightarrow \infty} q^{\lambda} .
$$

We shall see that the $x^{\lambda}$ are coordinates of an extension of Minkowski space in the sense of Kaluza and Klein.

Not every space-time can be the limit of a noncommutative geometry. One can in fact define a Poisson structure on $\mathcal{A}_{0}$ by setting

$$
\{f, g\}=-i \lim _{\mu_{P} \rightarrow \infty} \mu_{P}^{2}[f, g] .
$$


In particular

$$
\left\{x^{\mu}, x^{\nu}\right\}=\lim _{\mu_{P} \rightarrow \infty} q^{\mu \nu} .
$$

The $q^{\mu \nu}$ cannot in the commutative limit tend to a set of functions on space-time since this would break Lorentz invariance. (Any tensor except the metric breaks Lorentz invariance.) We conclude quite generally then that space-time must be of dimension greater than four if $q^{\mu \nu}$ does not identically vanish. Since there are 6 independent $q^{\mu \nu}$ in the commutative limit one would expect a space of at least 10 dimensions unless extra conditions are imposed. We shall avoid here the question of the physical significance of the extra dimensions. We refer to Madore \& Mourad (1996a) for a discussion of KaluzaKlein theory within the context of noncommutative geometry and to Dubois-Violette et al. (1996a) for a general discussion of the commutative limits of noncommutative algebras.

We now turn to the Planck mass as a universal cut-off. Let $T_{\mu \nu}^{(0)}$ be the bare energymomentum tensor, including quantum corrections, of some field theory on space-time, Choose some separation of $T_{\mu \nu}^{(0)}$ into a divergent part $T_{\mu \nu}^{\left(\mu_{P}\right)}$ and a regular part $T_{\mu \nu}^{(\mathrm{Reg})}$ which would remain finite if one were to let $\mu_{P} \rightarrow \infty$. Implicit in what follows is the assumption that the decomposition can be made so that the singular part is in some sense universal and independent of the particular (physically reasonable) field theory one starts with. We write then

$$
T_{\mu \nu}^{(0)}=T_{\mu \nu}^{\left(\mu_{P}\right)}+T_{\mu \nu}^{(\mathrm{Reg})}
$$

Denote by $\langle O\rangle_{0}$ the vacuum-expectation value of an operator $O$. Then in a quasiclassical approximation, considering the gravitational field as classical, one can write the Einstein field equations as

$$
G_{\mu \nu}=-\mu_{P}^{-2}\left(\left\langle T_{\mu \nu}^{\left(\mu_{P}\right)}\right\rangle_{0}+\left\langle T_{\mu \nu}^{(\mathrm{Reg})}\right\rangle_{0}\right)
$$

We shall be here interested in the divergent part of $T_{\mu \nu}^{(0)}$ and we shall neglect the regular term. The field equations become then

$$
G_{\mu \nu}=-\mu_{P}^{-2}\left\langle T_{\mu \nu}^{\left(\mu_{P}\right)}\right\rangle_{0}
$$

This equation is quite unsatisfactory. One would like to replace it by an operator equation of the form

$$
G_{\mu \nu}=-\mu_{P}^{-2} T_{\mu \nu}^{\left(\mu_{P}\right)}
$$

such that

$$
G_{\mu \nu}^{(\infty)}=\lim _{\mu_{P} \rightarrow \infty} G_{\mu \nu}
$$

is non-vanishing in order to produce a gravitational field which acts as a regulator but such that

$$
\left\langle G_{\mu \nu}^{(\infty)}\right\rangle_{0}=0
$$

so that the regularizing gravitational field is not classically observable. In general however it is reasonable to assume that a divergence gives rise to a gravitational field and with Equation (2.5) or (2.6) one can define the left arrow of the diagram

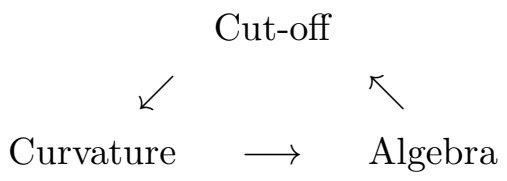


The idea in introducing the noncommutative structure is to eliminate points on small length scales. If this is achieved to within $\mu_{P}^{-1}$ then one would expect that the algebraic structure introduces a cut-off of order $\mu_{P}$. This is the right arrow of the above diagram. It has been discussed, for example, by Doplicher et al. The bottom arrow was discussed in the Introduction. It summerizes the argument that a strong classical or quantum gravitational field leads to a noncommutative version of space-time.

To give more substance to this qualitative argument we examine the following diagram:

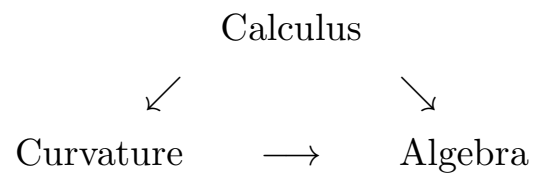

We shall argue that it can in fact be used to define the bottom arrow, the same as in (2.9). The right arrow is a mathematical triviality; it gives a relation between a differential calculus (to be defined below) over an algebra and the algebra itself. We shall argue that to a certain extent a differential calculus determines uniquely a linear connection in the commutative limit. The uniqueness will allow us to invert the left arrow. We can claim then that curvature gives rise not only to a noncommutative algebra but also to an associated differential calculus and as a corollary we have also defined the bottom arrow. To the extent that this can be done we hope to use the construction of the curvature in Diagram (2.10) to replace the Equations (2.5) or (2.6). That is, instead of trying to use the field equations to deduce the curvature directly from the divergent part $T_{\mu \nu}^{\left(\mu_{P}\right)}$ of $T_{\mu \nu}^{(0)}$ we would like to envisage the possibility that it can be indirectly inferred from the structure of the algebra which gives rise to $T_{\mu \nu}^{\left(\mu_{P}\right)}$. Since the differential calculus is not unique we cannot claim that the curvature depends only on the right-hand side of (1.1). That is, although the non-vanishing Planck mass gives rise to commutation relations, the left-hand side of (2.5) does not depend only on $\mu_{P}$. Were this the case then (2.5) would become an eigenvalue equation yielding the mass spectrum in units of $\mu_{P}$.

\section{Differential calculi in general}

From the mathematical point of view a gravitational field is a rule for displacing vectors and covectors and so we must have a definition of these objects in the noncommutative case. We shall see that there are certain problems associated with the definition of a vector but the noncommutative generalization of a covector or differential form (Connes $1986,1994)$ is quite satisfactory. A set of differential forms, with the associated exterior derivative, is called a differential calculus. The main problem as far as physics is concerned is the fact that there is no a priori unique way of defining a differential calculus over an arbitrary algebra. In this section we shall give the general definitions and illustrate them with simple examples. In the next section we shall more specifically define differential calculi over the algebras defined by relations of the form (1.1).

One of the characteristics of a noncommutative geometry is the lack of a welldefined notion of localization. This is to be expected since there are no points. In the particular case of an algebra of functions on a space one can speak of a function or element of the algebra as being localized near a given point. Functions can be chosen whose support is contained in an arbitrarily small region around the point. Vectors and covectors can be studied in a given region of space without due attention to whether or not their definition can be extended everywhere on the space. Noncommutative 
geometry on the other hand is essentially global. When studying a smooth space from the point of view of noncommutative geometry the algebra is the algebra of all smooth functions. By vectors and covectors we mean therefore in this case globally defined vector fields and covector fields.

There is a natural noncommutative generalization of a vector (field). To see this we first consider the commutative case. Let $X^{i}$ be the components of a vector and let $f$ be a smooth function. Then one can form the derivative $X^{i} \partial_{i} f$ of $f$ in the direction $X^{i}$. It is again a smooth function. That is, one can consider $X=X^{i} \partial_{i}$ as a linear map from the algebra of smooth functions into itself which satisfies the Leibniz rule: $X(f g)=(X f) g+f X g$. A map of this type of any (associative) algebra into itself is called a derivation. So what we have shown is that to each vector field on a smooth manifold one can associate a derivation of the algebra of smooth functions on the manifold. It is easy to convince oneself that the converse is true. We have therefore a natural generalization of a vector field in the noncommutative case. It is a derivation of the algebra. Notice that if $X$ is a derivation then so is $f X$ for any smooth function $f$; the derivations form a left module over the algebra.

As a simple noncommutative example it is instructive to consider again the algebra $M_{2}$ of complex $2 \times 2$ matrices. Let $\lambda_{i}$ be the Pauli matrices, chosen antihermitian and let $f$ be an arbitrary matrix. Then it is easy to see that the maps $e_{i}$ defined by

$$
e_{i} f=\left[\lambda_{i}, f\right]
$$

are derivations om $M_{2}$. Since $\lambda_{i}$ is antihermitian the derivation is real; the matrix $e_{i} f$ is hermitian whenever $f$ is. The Leibniz rule is the Jacobi identity. It can be shown that the most general derivation of $M_{2}$ is of the form $X=X^{i} e_{i}$ where the $X^{i}$ are complex numbers. The set of derivations form a vector space of dimension 3 . This example serves to illustrate the fact that in the noncommutative case a derivation cannot be multiplied from the left by an arbitrary element of the algebra; the derivations do not form a left module over the algebra. For this reason one usually tries to work as much as possible in noncommutative geometry with the generalization of covectors or differential forms.

Consider a smooth manifold and let $A_{i}$ be the components of a (smooth) covariant vector (field). We shall write it as $A=A_{i} d x^{i}$ using a set of basis elements $d x^{i}$ and so written we shall refer to it as a 1-form. A 2 -form is an antisymmetric 2-index covariant tensor $F_{i j}$ which we write as

$$
F=\frac{1}{2} F_{i j} d x^{i} d x^{j}
$$

using the product of the basis elements. This product is antisymmetric:

$$
d x^{i} d x^{j}=-d x^{j} d x^{i}
$$

but otherwise has no relations. Higher-order forms can be defined as arbitrary linear combination of products of 1 -forms. A $p$-form can be thus written as

$$
\alpha=\frac{1}{p !} \alpha_{i_{1} \cdots i_{p}} d x^{i_{1}} \cdots d x^{i_{p}} .
$$

The coefficients $\alpha_{i_{1} \cdots i_{p}}$ are smooth functions and completely antisymmetric in the $p$ indices.

Let $\mathcal{A}$ be the algebra of smooth complex-valued functions on a smooth manifold. We define $\Omega^{0}(\mathcal{A})=\mathcal{A}$ and for each $p$ we write the vector space of $p$-forms as $\Omega^{p}(\mathcal{A})$. Each $\Omega^{p}(\mathcal{A})$ depends obviously on the algebra $\mathcal{A}$ and, what is also obvious and very 
important, it can be multiplied both from the left and the right by the elements of $\mathcal{A}$. It is easy to see that $\Omega^{p}(\mathcal{A})=0$ for all $p$ greater than the dimension of the manifold. We define $\Omega^{*}(\mathcal{A})$ to be the set of all $\Omega^{p}(\mathcal{A})$. We have seen that $\Omega^{*}(\mathcal{A})$ has a product. It is a graded commutative algebra. It can be written as a sum

$$
\Omega^{*}(\mathcal{A})=\Omega^{+}(\mathcal{A}) \oplus \Omega^{-}(\mathcal{A})
$$

of even forms and odd forms. The algebra $\mathcal{A}$ is a subalgebra of $\Omega^{+}(\mathcal{A})$.

Let $f$ be a function, an element of the algebra $\mathcal{A}=\Omega^{0}(\mathcal{A})$. We define a map $d$ from $\Omega^{p}(\mathcal{A})$ into $\Omega^{p+1}(\mathcal{A})$ by the rules

$$
d f=\partial_{i} f d x^{i}, \quad d^{2}=0 .
$$

It takes odd (even) forms into even (odd) ones. From the rules we find that

$$
d A=d\left(A_{i} d x^{i}\right)=\frac{1}{2}\left(\partial_{i} A_{j}-\partial_{j} A_{i}\right) d x^{i} d x^{j}=F
$$

if we set

$$
F_{i j}=\partial_{i} A_{j}-\partial_{j} A_{i}
$$

From the second rule we have

$$
d F=0
$$

It is easy to see that if $\alpha$ is a $p$-form and $\beta$ is a $q$-form then

$$
\alpha \beta=(-1)^{p q} \beta \alpha, \quad d(\alpha \beta)=(d \alpha) \beta+(-1)^{p} \alpha d \beta .
$$

The couple $\left(\Omega^{*}(\mathcal{A}), d\right)$ is called a differential algebra or a differential calculus over $\mathcal{A}$. The algebra $\mathcal{A}$ need not be commutative and $\Omega^{*}(\mathcal{A})$ need not be graded commutative. Over each algebra $\mathcal{A}$, be it commutative or not, there can exist a multitude of differential calculi. As a simple example we define what is known as the universal differential calculus $\left(\Omega_{u}^{*}(\mathcal{A}), d_{u}\right)$ over a commutative algebra $\mathcal{A}$ of functions. We set, as always, $\Omega_{u}^{0}(\mathcal{A})=\mathcal{A}$ and for each $p \geq 1$ we define $\Omega_{u}^{p}(\mathcal{A})$ to be the set of $p$-point functions which vanish when any two points coincide. It is obvious that $\Omega_{u}^{p}(\mathcal{A}) \neq 0$ for all $p$. There is a map $d$ from $\Omega_{u}^{p}(\mathcal{A})$ into $\Omega_{u}^{p+1}(\mathcal{A})$ given in the lowest order by

$$
\left(d_{u} f\right)(x, y)=f(y)-f(x) .
$$

In higher orders it is given by a similar sort of alternating sum defined so that $d_{u}^{2}=0$. The algebra $\Omega_{u}^{*}(\mathcal{A})$ is not graded commutative. It is however defined for arbitrary functions, not necessarily smooth, and it has a straightforward generalization for arbitrary algebras, not necessarily commutative. To explain the qualifier 'universal' let $\left(\Omega^{*}(\mathcal{A}), d\right)$ be any other differential calculus over $\mathcal{A}$, for example the usual de Rham differential calculus constructed above. Then there is a unique $d_{u}$-homomorphism $\phi$

$$
\Omega_{u}^{*}(\mathcal{A}) \stackrel{\phi}{\longrightarrow} \Omega^{*}(\mathcal{A})
$$

of $\Omega_{u}^{*}(\mathcal{A})$ into $\Omega^{*}(\mathcal{A})$. It is given by

$$
\phi(f)=f, \quad \phi\left(d_{u} f\right)=d f .
$$

If we choose a coordinate system and expand the function $f(y)$ about the point $x$,

$$
f(y)=f(x)+\left(y^{i}-x^{i}\right) \partial_{i} f+\cdots,
$$


we see that the map $\phi$ is given by

$$
\phi\left(y^{i}-x^{i}\right)=d x^{i}
$$

and that it annihilates any 1 -form $f(x, y) \in \Omega_{u}^{1}(\mathcal{A})$ which is second order in $x-y$. One such form is $f d_{u} g-d_{u} g f$, given by

$$
\left(f d_{u} g-d_{u} g f\right)(x, y)=-(f(y)-f(x))(g(y)-g(x)) .
$$

It does not vanish in $\Omega_{u}^{1}(\mathcal{A})$ but its image in $\Omega^{1}(\mathcal{A})$ under $\phi$ is equal to zero. For further definitions we refer, for example, to the book by Connes (1994). See also Madore (1995).

What distinguishes the de Rham differential calculus is the fact that it is based on derivations. The relation between $d$ and $\partial_{i}$ is given by

$$
d f\left(\partial_{i}\right)=\partial_{i} f
$$

Notice that in particular this formula defines $d x^{i}$. The derivations form a vector space (the tangent space) at each point, and the above equation defines $d f$ as an element of the dual vector space (the cotangent space) at the same point. In the examples we shall consider there are no points but the vector spaces of derivations are still ordinary finitedimensional vector spaces. Over an arbitrary algebra which has derivations one can always define in exactly the same manner differential calculi based on the derivations. These algebras have thus at least two, quite different, differential calculi, the universal one and the one based on the set of all derivations.

To illustrate the notion of forms in the noncommutative case we return again to the algebra $M_{2}$ and show how to define $d \lambda_{i}$ using the derivations (Dubois-Violette 1988, Dubois-Violette et al. 1989). Let $f$ be an element of $M_{2}$ and introduce the $S U_{2}$ structure constants $C^{i}{ }_{j k}$. We write them in this abstract form so that all the formulae we write have immediate generalizations to the algebra $M_{n}$ of $n \times n$ matrices provided we replace $S U_{2}$ by $S U_{n}$. The straightforward extension of (3.4) can be written

$$
d \lambda_{j}\left(e_{i}\right)=\left[\lambda_{i}, \lambda_{j}\right]=C^{k}{ }_{i j} \lambda_{k} .
$$

The set of $d \lambda_{i}$ constitutes a system of generators of $\Omega^{1}\left(M_{2}\right)$ as a left or right module but it is not a convenient one. For example $\lambda_{i} d \lambda_{j} \neq d \lambda_{j} \lambda_{i}$. There is a better system of generators completely characterized by the equations

$$
\theta^{i}\left(e_{j}\right)=\delta_{j}^{i}
$$

We refer to the $\theta^{i}$ as a frame or Stehbein. They are related to the $d \lambda_{i}$ by the equations

$$
d \lambda_{i}=-C^{j}{ }_{i k} \lambda_{j} \theta^{k}, \quad \theta^{i}=\lambda_{j} \lambda^{i} d \lambda^{j} .
$$

They are the matrix analogue of the dual basis of the 1-forms. We have raised and lowered indices here using the Killing metric on the Lie algebra of $S U_{2}$. Because of the relation (3.6) we can write the differential of any element $f$ of $M_{2}$ as

$$
d f=e_{i} f \theta^{i}
$$

Also because of the relation (3.6) we have

$$
\theta^{i} \theta^{j}=-\theta^{j} \theta^{i}, \quad \lambda_{i} \theta^{j}=\theta^{j} \lambda_{i} .
$$


The product is the product in $\Omega^{*}\left(M_{2}\right)$; it is not in general antisymmetric, Since the $\theta^{i}$ commute with the elements of $M_{2}$ we can identify $\Omega^{1}\left(M_{2}\right)$ with the tensor product of $M_{2}$ and the dual $\wedge^{1}$ to the Lie algebra of $S U_{2}$ :

$$
\Omega^{1}\left(M_{2}\right)=M_{2} \otimes \bigwedge^{1}
$$

Technically speaking, $\Omega^{1}\left(M_{2}\right)$ is a free left (or right) $M_{2}$-module of rank equal to the dimension of the Lie algebra of $S U_{2}$. The geometry of $M_{2}$ is similar in this respect to the geometry of a parallelizable manifold.

From the generators $\theta^{i}$ we can construct a 1 -form

$$
\theta=-\lambda_{i} \theta^{i}
$$

in $\Omega^{1}\left(M_{2}\right)$ which satisfies the equation

$$
d \theta+\theta^{2}=0
$$

It follows directly from the definitions that the exterior derivative $d f$ of an element of $M_{2}$ can be written in terms of a commutator with $\theta$ :

$$
d f=-[\theta, f]
$$

It follows that as a bimodule $\Omega^{1}\left(M_{2}\right)$ is generated by one element.

As a last example we consider a differential calculus over the algebra of functions on a space of 2 points. We cannot use the construction based on derivations since this algebra has none. We write the algebra $M_{2}$ as the direct sum

$$
M_{2}=M_{2}^{+} \oplus M_{2}^{-}
$$

of the diagonal matrices $M_{2}^{+}$and the off-diagonal matrices $M_{2}^{-}$. We saw in the introduction that we can identify the algebra of functions on the space of 2 points with $M_{2}^{+}$. We set for each $p \geq 0$

$$
\Omega^{2 p}\left(M_{2}^{+}\right)=M_{2}^{+}, \quad \Omega^{2 p+1}\left(M_{2}^{+}\right)=M_{2}^{-} .
$$

We choose an arbitrary (antihermitian)element $\theta$ in $M_{2}^{-}$and we define the differential $d$ by the formula (3.13) but with a graded bracket:

$$
[\alpha, \beta]=\alpha \beta-(-1)^{|\alpha||\beta|} \beta \alpha .
$$

Then we see immediately that if $\theta^{2}=-1$ then $d^{2}=0$. One can easily show that this differential calculus is in fact the universal differential calculus over $M_{2}^{-}$. Although the space is rather trivial the differential calculus has non-trivial entries in all dimensions. We can identify however all even and odd forms and write $\Omega^{*}\left(M_{2}^{+}\right)=M_{2}$. A formula similar to (3.13) can be written in ordinary geometry using the Dirac operator instead of $\theta$ and a differential calculus based on an appropriate generalization of (3.13) can we defined for a large class of algebras (Connes 1994).

To form tensors one must be able to define tensor products, for example the tensor product $\Omega^{1}(\mathcal{A}) \otimes_{\mathcal{A}} \Omega^{1}(\mathcal{A})$ of $\Omega^{1}(\mathcal{A})$ with itself. We have here written in subscript the algebra $\mathcal{A}$. This indicates the fact that we identify $\xi f \otimes \eta$ with $\xi \otimes f \eta$ for every element $f$ of the algebra and it means that one must be able to multiply the elements of $\Omega^{1}(\mathcal{A})$ on the left and on the right by the elements of the algebra $\mathcal{A}$. If $\mathcal{A}$ is commutative of course these two operations are equivalent. When $\mathcal{A}$ is an algebra of functions this left/right linearity is equivalent to the property of locality. It means that the product 
of a function with a 1-form at a point is again a 1-form at the same point, a property which distinguishes the ordinary product from other, non-local, products such as the convolution. In the noncommutative case there are no points and locality cannot be defined; it is replaced by the property of left and/or right linearity with respect to the algebra.

The construction of the differential calculus over the algebra $M_{2}$ which we gave above relies on two properties of the derivations $e_{i}$. First and most important these derivations are such that from the identity $e_{i} f=0$ follows that $\mathrm{f}$ must be proportional to the identity matrix. Such elements are the noncommutative generalization of constant functions. We have then 'sufficient' derivations. The second property which we would have used if we had entered into the details of the construction of the higherorder forms is that the derivations form a Lie algebra. It is in fact the Lie algebra of all derivations of $M_{2}$. The first property is essential but this second property can be relaxed. One can construct differential calculi over an algebra $\mathcal{A}$ based on sets of derivations which form a proper Lie subalgebra of the Lie algebra of all derivations or which indeed have no special Lie-algebra property. To close this section we outline such a construction.

Let $\mathcal{A}$ be any algebra and let $\left(\Omega_{u}^{*}(\mathcal{A}), d_{u}\right)$ be the universal differential calculus over $\mathcal{A}$. Every other differential calculus can be considered as a quotient of the universal one. For this construction we refer to the book by Connes (1994). See also Madore (1995). Let $\left(\Omega^{*}(\mathcal{A}), d\right)$ be another differential calculus over $\mathcal{A}$. Then there exists a unique $d_{u}$-homomorphism $\phi$ of $\Omega_{u}^{*}(\mathcal{A})$ onto $\Omega^{*}(\mathcal{A})$. It is given by

$$
\phi\left(d_{u} f\right)=d f .
$$

The restriction $\phi_{p}$ of $\phi$ to each $\Omega_{u}^{p}$ is defined by

$$
\phi_{p}\left(f_{0} d_{u} f_{1} \cdots d_{u} f_{p}\right)=f_{0} d f_{1} \cdots d f_{p} .
$$

Consider a given algebra $\mathcal{A}$ and suppose that we know how to construct an $\mathcal{A}$ module $\Omega^{1}(\mathcal{A})$ and an application

$$
\mathcal{A} \stackrel{d}{\longrightarrow} \Omega^{1}(\mathcal{A})
$$

Then using (3.14) there is a method of constructing $\Omega^{p}(\mathcal{A})$ for $p \geq 2$ as well as the extension of the differential. Since we know $\Omega_{u}^{1}(\mathcal{A})$ and $\Omega^{1}(\mathcal{A})$ we can suppose that $\phi_{1}$ is given. We must construct $\Omega^{2}(\mathcal{A})$. We shall choose $\Omega^{2}(\mathcal{A})$ to be the largest set of 2 -forms consistent with the constraints on $\Omega^{1}(\mathcal{A})$. From general arguments we know that it can be written in the from

$$
\Omega^{2}(\mathcal{A})=\Omega_{u}^{2}(\mathcal{A}) / \mathcal{K}
$$

for some bimodule $\mathcal{K}$. Since we wish to have for every 1 -form $\xi_{u}$ in $\Omega_{u}^{1}(\mathcal{A})$

$$
d \phi_{1}\left(\xi_{u}\right)=\phi_{2}\left(d_{u} \xi_{u}\right)
$$

we see that $\mathcal{K}$ must contain $d_{u} \operatorname{Ker} \phi_{1}$. We can choose $\mathcal{K}$ to be the bimodule generated by $d_{u} \operatorname{Ker} \phi_{1}$. Let $\phi_{2}$ be the projection of $\Omega_{u}^{2}(\mathcal{A})$ onto $\Omega^{2}(\mathcal{A})$. The product of two elements $\xi$ and $\eta$ in $\Omega^{1}(\mathcal{A})$ is defined by choosing two inverse images $\xi_{u}$ and $\eta_{u}$ in $\Omega_{u}^{1}(\mathcal{A})$ and projecting their product onto $\Omega^{2}(\mathcal{A})$ :

$$
\xi \eta=\phi_{2}\left(\xi_{u} \otimes \eta_{u}\right)
$$


This procedure can be continued by iteration to arbitrary order in $p$.

To initiate the above construction we define the 1-forms using a set of derivations. For each integer $n$ let $\lambda_{i}$ be a set of $n$ linearly independent antihermitian elements of $\mathcal{A}$ and proceed as in (3.5) except that we can now write only

$$
d \lambda_{j}\left(e_{i}\right)=\left[\lambda_{i}, \lambda_{j}\right]
$$

To complete the construction we must postulate the existence of a set of 1-forms $\theta^{i}$ which satisfy (3.6). In particular cases this existence can be proven by explicit calculation. Notice that the integer $n$ need not be equal to the number of generators of the algebra but as a left or right module $\Omega^{1}(\mathcal{A})$ is free of rank $n$, that is, it has the $\theta^{i}$ as a basis over the algebra. This is an essential property of parallelizable manifolds of dimension $n$. Here the property can be made to hold for every integer $n$, provided of course that the algebra $\mathcal{A}$ is noncommutative. If a commutative limit is taken then the basis will be singular if $n$ is not equal to the dimension of the resulting manifold. We refer to Dimakis \& Madore (1996) for an example of this.

Because of the commutation relations of the algebra or, equivalently, because of the kernel of $\phi_{1}$ in the quotient (3.15) the $\theta^{\alpha}$ satisfy in general commutation relations. In one important case which we shall consider they anticommute. Because the 2-forms are generated by products of the $\theta^{i}$ one has

$$
d \theta^{i}=-\frac{1}{2} C^{i}{ }_{j k} \theta^{j} \theta^{k} .
$$

The structure elements $C^{i}{ }_{j k}$ can be $a$ priori arbitrary elements of the algebra.

Although we have made no explicit hypothesis concerning the $\lambda_{i}$ except that they be antihermitian and linearly independent, the existence of the basis $\theta^{i}$ places severe restrictions on them. In fact one can show that there must exist elements $P^{l m}{ }_{j k}, F_{j k}^{i}$ and $K_{j k}$ in the center of the algebra such that

$$
2 \lambda_{l} \lambda_{m} P^{l m}{ }_{j k}-\lambda_{i} F^{i}{ }_{j k}-K_{j k}=0 .
$$

The $P^{l m}{ }_{j k}$ defines a projection of $\Omega^{1}(\mathcal{A}) \otimes \Omega^{1}(\mathcal{A})$ onto $\Omega^{2}(\mathcal{A})$. If it vanishes then so does $\Omega^{2}(\mathcal{A})$. From the associative rule for the algebra follow conditions on the coefficients, Since the frame is determined by the derivations it is to be expected that the structure elements are determined by the coefficients in (3.17):

$$
C^{i}{ }_{j k}=F^{i}{ }_{j k}-2 \lambda_{l} P^{(l i)}{ }_{j k} \text {. }
$$

We refer to Madore \& Mourad (1996b) for details.

\section{Differential calculi over fuzzy space-time}

The general formalism for the construction of differential calculi can be applied in particular to the algebra which we introduced in Section 2. Suppose as a first approximation that (2.1) is satisfied and that the matrix $q^{\mu \nu}$ has an inverse $q_{\lambda \mu}^{-1}$ :

$$
q_{\lambda \mu}^{-1} q^{\mu \nu}=\delta_{\lambda}^{\nu}
$$

We shall use this inverse to lower the indices of the generators $q^{\mu}$ :

$$
\tilde{q}_{\lambda}=\mu_{P}^{2} q_{\lambda \mu}^{-1} q^{\mu} .
$$


A natural choice of $n$ is $n=4$ and a natural choice of $\lambda_{\mu}$ is given by

$$
\lambda_{\mu}=-i \tilde{q}_{\mu}
$$

The associated derivations defined in Section 2 satisfy then

$$
e_{\mu} q^{\lambda}=\delta_{\mu}^{\lambda}
$$

and it follows that

$$
\left[e_{\mu}, e_{\nu}\right]=0 \text {. }
$$

Notice that if $f$ is an element of $\mathcal{A}_{\mu_{P}}$ such that $e_{\mu} f=0$ then we can only conclude that $f$ is an arbitrary function of the $q^{\mu \nu}$. We cannot conclude that it is proportional to the identity. We accept this fact since we regard the non-trivial center as something which is to be eventually eliminated. With proper conditions the center can be regarded as a smooth manifold and treated using ordinary differential geometry.

From (4.2) it follows that

$$
\theta^{\lambda}=d q^{\lambda}, \quad \theta=i \tilde{q}_{\lambda} d q^{\lambda}
$$

from which we deduce that

$$
P_{\rho \sigma}^{\mu \nu}=\frac{1}{2}\left(\delta_{\rho}^{\mu} \delta_{\sigma}^{\nu}-\delta_{\rho}^{\nu} \delta_{\sigma}^{\mu}\right), \quad F_{\mu \nu}^{\lambda}=0, \quad K_{\mu \nu}=i \mu_{P}^{2} q_{\mu \nu}^{-1} .
$$

From the commutation relations one finds that the $\theta^{\lambda}$ anticommute. One sees from their definition (3.16), or from (3.18), that the structure elements vanish:

$$
C_{\mu \nu}^{\lambda}=0 .
$$

We shall see below in Section 5 that this implies that as in the ordinary case the only torsion-free metric connection is the flat one; the space-time is a noncommutative version of Minkowski space. To find non-trivial gravitational fields we must change the differential calculus. We would like to maintain the algorithm outlined in Section 2. We must therefore either change the Ansatz (4.1) or the structure of the algebra $\mathcal{A}_{\mu_{P}}$.

We shall consider first-order perturbations of the $\lambda_{\mu}$ defined by (4.1). Introduce four arbitrary 'small' elements $f^{\lambda}$ of $\mathcal{A}$ and define

$$
\tilde{f}_{\lambda}=\mu_{P}^{2} q_{\lambda \mu}^{-1} f^{\mu}
$$

Then the elements

$$
\lambda_{\mu}^{\prime}=-i\left(\tilde{q}_{\mu}+\tilde{f}_{\mu}\right)
$$

are 'near' to (4.1). In general, unless the condition (3.17) is satisfied, $\Omega^{2}(\mathcal{A})=0$ and the curvature will vanish. Impose the condition (3.17) and let $P_{(1) \mu \nu}^{\rho \sigma}, F_{(1) \mu \nu}^{\lambda}$ and $K_{(1) \mu \nu}$ be the first-order perturbations respectively of the coefficients. By simple dimensional arguments one can argue that $P_{(1)}^{\mu \nu} \rho \sigma$ must vanish. In fact it must tend to zero when the Planck mass tends to infinity but on the other hand it is without dimension and therefore cannot depend on the Planck mass. Therefore we set

$$
P_{(1) \rho \sigma}^{\mu \nu}=0
$$

Using (4.5) we find that the linearization of (3.17) yields the equation

$$
\left[\tilde{q}_{\mu}, \tilde{f}_{\nu}\right]-\left[\tilde{q}_{\nu}, \tilde{f}_{\mu}\right]=i F_{(1) \mu \nu}^{\lambda} \tilde{q}_{\lambda}-K_{(1) \mu \nu}
$$


Let $k_{\mu}$ be an arbitrary 'small' 4-vector with the dimension of mass. Then a solution is given by

$$
\tilde{f}_{\lambda}=k_{\mu} q^{\mu} \tilde{q}_{\lambda}
$$

and

$$
F_{(1) \mu \nu}^{\lambda}=k_{[\mu} \delta_{\nu]}^{\lambda}+2 q_{\mu \nu}^{-1} q^{\lambda \sigma} k_{\sigma}, \quad K_{(1) \mu \nu}=0 .
$$

The corresponding frame is given by

$$
\theta^{\prime \lambda}=\left(1-k_{\rho} q^{\rho}\right) d q^{\lambda}+q^{\lambda \rho} k_{\rho} q_{\mu \sigma}^{-1} q^{\sigma} d q^{\mu} .
$$

It will generate a new differential calculus $\Omega^{\prime *}(\mathcal{A})$ which will be in general different from $\Omega^{*}(\mathcal{A})$. Using $(3.17)$ or $(3.18)$ we find that

$$
C_{(1) \mu \nu}^{\lambda}=F_{(1) \mu \nu}^{\lambda} .
$$

We shall see below that the modified calculus admits a gravitational field.

It is interesting also to maintain the Ansatz (4.1) but perturb the condition (2.1) which restricted the algebra $\mathcal{A}_{\mu_{P}}$. We introduce 6 'small' elements $q_{(1)}^{\mu \nu}$ of $\mathcal{A}_{\mu_{P}}$ and define

$$
q^{\prime \mu \nu}=q^{\mu \nu}+q_{(1)}^{\mu \nu} .
$$

We have then

$$
q_{\mu \nu}^{\prime-1}=q_{\mu \nu}^{-1}+q_{\mu \rho}^{-1} q_{\nu \sigma}^{-1} q_{(1)}^{\rho \sigma}
$$

Define

$$
\lambda_{\mu}^{\prime}=\lambda_{\mu}=-i \tilde{q}_{\mu}
$$

We set

$$
\left[q^{\lambda}, q^{\prime \mu \nu}\right]=\left[q^{\lambda}, q_{(1)}^{\mu \nu}\right]=i \mu_{P}^{-1} q_{(1)}^{\lambda \mu \nu} .
$$

The simplest generalization of the condition (2.1) is to suppose that $q_{(1)}^{\lambda \mu \nu}$ lies in the center of $\mathcal{A}_{\mu_{P}}$. This is the extended model of Doplicher et al. If we impose this condition we can choose

$$
q_{(1)}^{\mu \nu}=-\mu_{P}^{-1} q_{(1)}^{\lambda \mu \nu} \tilde{q}_{\lambda}
$$

We have then

$$
e_{\mu}^{\prime} q^{\nu}=e_{\mu} q^{\nu}=\delta_{\mu}^{\nu}+\mu_{P}^{-1} q_{\mu \rho}^{-1} q_{(1)}^{\nu \rho \sigma} \tilde{q}_{\sigma}
$$

Using (4.5) we find that the linearization of (3.17) yields now the solution

$$
F_{(1) \rho \sigma}^{\lambda}=\mu_{P} q_{\rho \mu}^{-1} q_{\sigma \nu}^{-1}\left(q_{(1)}^{\lambda \mu \nu}-q_{(1)}^{\mu \nu \lambda}+q_{(1)}^{\nu \mu \lambda}\right) .
$$

The corresponding frame is given by

$$
\theta^{\prime \lambda}=d q^{\lambda}-\mu_{P}^{-1} q_{\mu \rho}^{-1} q_{(1)}^{\lambda \rho \sigma} \tilde{q}_{\sigma} d q^{\mu} .
$$

From (4.14) we see that this leads again to a rather trivial but nonvanishing gravitational field. 


\section{$5 \quad$ Metrics and linear connections}

The definition of a connection as a covariant derivative was given an algebraic form in the Tata lectures by Koszul (1960). We shall use here the expressions 'connection' and 'covariant derivative' synonymously. We shall consider it as a rule which to a covector $\xi=\xi_{\lambda} d x^{\lambda}$ associates its covariant derivative $D \xi=D_{\mu} \xi_{\nu} d x^{\mu} \otimes d x^{\nu}$. For a detailed description of the definition of a linear connection in noncommutative geometry we refer to the article by Dubois-Violette et al. (1996b). We shall here make use of the basis elements $\theta^{\alpha}$. That is, since $\Omega^{1}(\mathcal{A})$ is a free module a covariant derivative can be defined by its action on these elements:

$$
D \theta^{\alpha}=-\omega^{\alpha}{ }_{\beta \gamma} \theta^{\beta} \otimes \theta^{\gamma} .
$$

The extension to arbitrary elements is given by the Leibniz rule. If $\xi=\xi_{\alpha} \theta^{\alpha}$ is an arbitrary 1-form then

$$
D \xi=d \xi_{\alpha} \otimes \theta^{\alpha}+\xi_{\alpha} D \theta^{\alpha} .
$$

The coefficients $\omega^{\alpha}{ }_{\beta \gamma}$ are elements of the algebra. Because of the identity $D\left(f \theta^{\alpha}\right)=$ $D\left(\theta^{\alpha} f\right)$ they cannot however be arbitrary elements. To see this we rewrite $\xi$ as $\xi=\theta^{\alpha} \xi_{\alpha}$ and we rewrite (5.2) as

$$
D \xi=\sigma\left(\theta^{\alpha} \otimes d \xi_{\alpha}\right)+\left(D \theta^{\alpha}\right) \xi_{\alpha} .
$$

The purpose of the map $\sigma$ is to place the differential to the left where is belongs while respecting the order of the terms. It is discussed in detail in Dubois-Violette et al. (1996). In the simple cases which we consider here it can be shown to be a simple transposition. We can conclude therefore that the coefficients $\omega^{\alpha}{ }_{\beta \gamma}$ must lie in the center of $\mathcal{A}_{\mu_{P}}$. This is not always the case.

One can define a metric by the condition

$$
g\left(\theta^{\alpha} \otimes \theta^{\beta}\right)=g^{\alpha \beta}
$$

where the coefficients $g^{\alpha \beta}$ are elements of the algebra. To be well defined on all elements of the tensor product $\Omega^{1}(\mathcal{A}) \otimes_{\mathcal{A}} \Omega^{1}(\mathcal{A})$ the metric must be bilinear and by the sequence of identities

$$
f g^{\alpha \beta}=g\left(f \theta^{\alpha} \otimes \theta^{\beta}\right)=g\left(\theta^{\alpha} \otimes \theta^{\beta} f\right)=g^{\alpha \beta} f
$$

one concludes that the coefficients must lie in the center of $\mathcal{A}$. This restriction plays an important role in restricting the admissible connections. In the commutative limit the $g^{\alpha \beta}$ cannot be functions of the coordinates. The Stehbein not only determines the differential calculus it determines also essentially the metric.

When $\sigma$ is a simple transposition the condition that $D$ be torsion-free becomes to first order the usual condition

$$
\omega_{\mu \nu}^{\lambda}-\omega_{\nu \mu}^{\lambda}=C_{\mu \nu}^{\lambda}
$$

The condition that a connection be metric is a straightforward generalization of the corresponding condition in the commutative case. Again when $\sigma$ is a simple transposition it becomes to first order the condition

$$
\omega_{\mu \nu}^{\lambda}+\omega_{\nu \mu}^{\lambda}=0 .
$$

The unique solution to these two conditions is given as usual by

$$
\omega_{\mu \nu}^{\lambda}=-\frac{1}{2}\left(C_{\nu \mu}^{\lambda}-C_{\mu}^{\lambda}{ }_{\nu}+C_{\nu \mu}{ }^{\lambda}\right) .
$$


The basic calculus admits therefore no connections with non-vanishing curvature. However the two perturbations which we have considered in Section 4 yield connections with non-vanishing albeit rather trivial curvature in the commutative limit.

There is no completely general satisfactory definition of the curvature of a linear connection in the noncommutative case. However the map $D$ can be extended by

$$
\Omega^{1}(\mathcal{A}) \otimes_{\mathcal{A}} \Omega^{1}(\mathcal{A}) \stackrel{D}{\longrightarrow} \Omega^{2}(\mathcal{A}) \otimes_{\mathcal{A}} \Omega^{1}(\mathcal{A})
$$

and one might be tempted to define curvature by

$$
D^{2} \theta^{\alpha}=-\frac{1}{2} R_{\beta \gamma \delta}^{\alpha} \theta^{\gamma} \theta^{\delta} \otimes \theta^{\beta}
$$

One can show that $D^{2}$ is left-linear,

$$
D^{2}\left(f \theta^{\alpha}\right)=f D^{2} \theta^{\alpha}
$$

but it is not in general right-linear:

$$
D^{2}\left(\theta^{\alpha} f\right) \neq\left(D^{2} \theta^{\alpha}\right) f
$$

A detailed discussion has been given by Dubois-Violette et al. (1996b). To lowest order we find the expression

$$
R_{\nu \rho \sigma}^{\mu}=\omega_{(1) \rho \tau}^{\mu} \omega_{(1) \sigma \nu}^{\tau}-\omega_{(1) \sigma \tau}^{\mu} \omega_{(1) \rho \nu}^{\tau}-\omega_{(1) \tau \nu}^{\mu} C_{(1) \rho \sigma}^{\mu}
$$

for the components of the curvature where the $\omega_{(1) \mu \nu}^{\lambda}$ are determined in terms of the $C_{(1) \mu \nu}^{\lambda}$ by (5.8). It is the 'Einstein tensor' which is obtained from this expression for the curvature which is to be placed on the left-hand side of Equation (2.5). We shall not pursue this further here since in order to make sense of the resulting equation an average must be performed over the coordinates $q^{\mu \nu}, q^{\lambda \mu \nu}$ of the extra dimensions.

\section{Acknowledgements}

The author would like to thank J. Mourad for interesting conversations.

\section{References}

Ackermann T., Tolksdorf J. 1996, A generalized Lichnerowicz formula, the Wodzicki Residue and Gravity, Jour. Geom. and Phys. (to appear).

Chamseddine A., Connes A. 1996, The spectral action principle (in preparation).

Connes A. 1986, Non-Commutative Differential Geometry, Publications of the Inst. des Hautes Etudes Scientifique. 62257.

- 1994, Noncommutative Geometry, Academic Press.

- 1996, Gravity coupled with matter and the foundation of non commutative geometry, IHES Preprint, hep-th/9603053.

Deser S. 1957, General Relativity and the Divergence Problem in Quantum Field Theory, Rev. Mod. Phys. 29417. 
Dimakis A., Madore J. 1996, Differential Calculi and Linear Connections, J. Math. Phys. (to appear).

Doplicher S., Fredenhagen K., Roberts, J.E. 1994, Spacetime quantization induced by classical gravity, Phys. Lett. B331 39.

- 1995, The Quantum Structure of Spacetime at the Planck Scale and Quantum Fields, Commun. Math. Phys. 172187.

Dubois-Violette M. 1988, Dérivations et calcul différentiel non-commutatif, C. R. Acad. Sci. Paris 307 Série I (1988) 403.

Dubois-Violette M., Kerner R., Madore J. 1989, Gauge bosons in a noncommutative geometry, Phys. Lett. B217 485; Classical bosons in a noncommutative geometry, Class. Quant. Grav. 61709.

- 1996a, Shadow of Noncommutativity, Preprint LPTHE Orsay 96/06.

Dubois-Violette M., Madore J., Masson T., Mourad J. 1996b, On Curvature in Noncommutative Geometry, J. Math. Phys. (to appear).

Frittelli S., Kozameh C.N., Newman E.T., Rovelli C., Tate R.S. 1996, Fuzzy spacetime from a null-surface version of GR, Pittsbourg Preprint.

Isham C.J., Salam A., Strathdee J. 1971, Infinity Suppression in Gravity-Modified Quantum Electrodynamics, Phys. Rev. D3 1805.

Kalau W., Walze M. 1995, Gravity, Non-Commutative Geometry and the Wodzicki Residue, Jour. Geom. and Phys. 16327.

Koszul J.L. 1960, Lectures on Fibre Bundles and Differential Geometry, Tata Institute of Fundamental Research, Bombay.

Madore J. 1988, Non-Commutative Geometry and the Spinning Particle, XI Warsaw Symposium on Elementary Particle Physics, Kazimierz, Poland; Kaluza-Klein Aspects of Noncommutative Geometry, Proceedings of the XVII International Conference on Differential Geometric Methods in Theoretical Physics, Chester.

- 1992, Fuzzy Physics, Annals of Physics 219187.

- 1995, An Introduction to Noncommutative Differential Geometry and its Physical Applications, Cambridge University Press.

Madore J., Mourad. J. 1995, On the origin of Kaluza-Klein structure, Phys. Lett. B359 43.

- 1996a, Noncommutative Kaluza-Klein Theory, Lecture given at the $5^{\text {th }}$ Hellenic School and Workshops on Elementary Particle Physics, hep-th/9601169.

- 1996b, Quantum Space-Time and Classical Gravity, Preprint, LPTHE Orsay, 95/56, gr-qc/9607060.

Mourad. J. 1995, Linear Connections in Non-Commutative Geometry, Class. Quant. Grav. 12965.

Snyder H.S. 1947, Quantized Space-Time, Phys. Rev. 7138.

't Hooft G. 1996 Quantization of point particles in (2+1)-dimensional gravity and spacetime discreteness, Class. Quant. Grav. 131023. 